\title{
Aberrant Right Ovarian Artery from Proximal Internal Iliac Artery in Uterine Artery Embolization Patient
}

\author{
Richard A. Reed ${ }^{1}$, Bruce McLucas ${ }^{2 *}$ \\ ${ }^{1}$ Department of Radiology, Huntington Memorial Hospital, Pasadena, USA \\ ${ }^{2}$ Department of Obstetrics and Gynecology, David Geffen School of Medicine at the University of California, \\ Los Angeles, USA \\ Email: *mclucas@ucla.edu
}

Received October 3, 2012; revised November 3, 2012; accepted November 15, 2012

\begin{abstract}
Some patients who undergo Uterine Artery Embolization (UAE) for symptomatic leiomyomata have collateral ovarian artery supply to the uterus. This typically occurs when the ipsilateral uterine artery is small or absent, and the ovarian artery supply to the uterus can lead to UAE failure. The authors present a case of a woman treated with UAE who had an atretic right uterine artery and an enlarged right ovarian artery supplying the fibroids. The ovarian artery arose from the proximal internal iliac artery. This rare variant vessel was embolized leading to eradication of the patient's symptoms. The success of this case highlights the need to evaluate for possible variant ovarian artery supply and to embolize these vessels in an attempt to prevent UAE failure.
\end{abstract}

Keywords: Uterine Artery Embolization; Fibroids; Minimally Invasive

\section{Introduction}

UAE has emerged as an effective and widely accepted treatment option for women with symptomatic uterine fibroids. Technical success rate approaches $100 \%$ but clinical success varies from 85\% - 94\% [1-4]. Collateral blood flow from an ovarian artery to the uterus can impede the desired infarction and lead to clinical failure. It has been shown that embolization of a hypertrophied ovarian artery that provides collateral flow to the uterus can lead to fibroid infarction [5].

The ovarian arteries have a characteristic tortuous course on angiography and most commonly arise from the anterior surface of the abdominal aorta just below the level of the renal arteries. Variations in the location of the ovarian artery origin have been reported including those that arise from the renal arteries, the suprarenal aorta or the sides of the abdominal aorta [6]. Less common origins have been reported including the inferior mesenteric artery [7] and the right external iliac artery [8].

Herein we report a case in which a woman with symptomatic uterine fibroids underwent UAE. There was an atretic right uterine artery and a hypertrophied aberrant ovarian artery arising from the proximal internal iliac artery. This vessel was embolized in an effort to maximize the chance for successful eradication of the

"Corresponding author. patient's fibroid related symptoms.

\section{Case Report}

A 45-year old female with three years of worsening menorrhagia and anemia due to symptomatic leiomyomata underwent bilateral UAE. The left internal iliac artery was catheterized with a 4-F Cobra-2 catheter (Glidecath, Terumo, Tokyo Japan). Following this a 2.7 French microcatheter (Progreat, Terumo, Tokyo, Japan) and 0.018 inch wire were coaxially advanced through the guiding catheter to catheterize the left uterine artery. Embolization with endpoint of near stasis of the left uterine artery was then achieved with two syringes of $500-700$ micron microspheres (Embospheres, Merit Medical, Salt Lake City). Following this the right internal iliac artery was catheterized with the same 4-F Cobra-2 catheter and an angiogram showed an atretic right uterine artery (Figure 1). This artery was catheterized with the coaxial 2.7 French microcatheter and the 0.018 inch wire. Embolization was achieved using one-eighth of a syringe of 500 - 700 micron microspheres.

The $4 \mathrm{~F}$ catheter was exchanged for a $5 \mathrm{~F}$ pigtail catheter and the tip of this catheter was advanced into the abdominal aorta just cephalad to the origin of the renal arteries to evaluate for possible hypertrophied ovarian artery supply to the fibroids. Abdominal aortogram showed an enlarged right ovarian artery with its typical corkscrew pattern arising from the origin of the right internal 
iliac artery (Figure 2). To get a better understanding of the amount of uterine artery supply that this aberrant ovarian artery contributed to the uterus, the ovarian artery was selectively catheterized with a $5 \mathrm{~F}$ Simmons 1 catheter.

The ovarian artery angiogram showed significant supply to the uterus (Figure 3). It was felt that if this artery was not embolized the fibroids would remain viable and the clinical symptoms would not resolve. The 2.7 French microcatheter in combination with a 0.018 inch wire was advanced coaxially through the 5F Simmons 1 catheter further into the ovarian artery. From this position embolization was performed with one syringe of 700 - 900 micron microspheres. At completion there was near stasis in the vessel. Subsequently the patient reported that

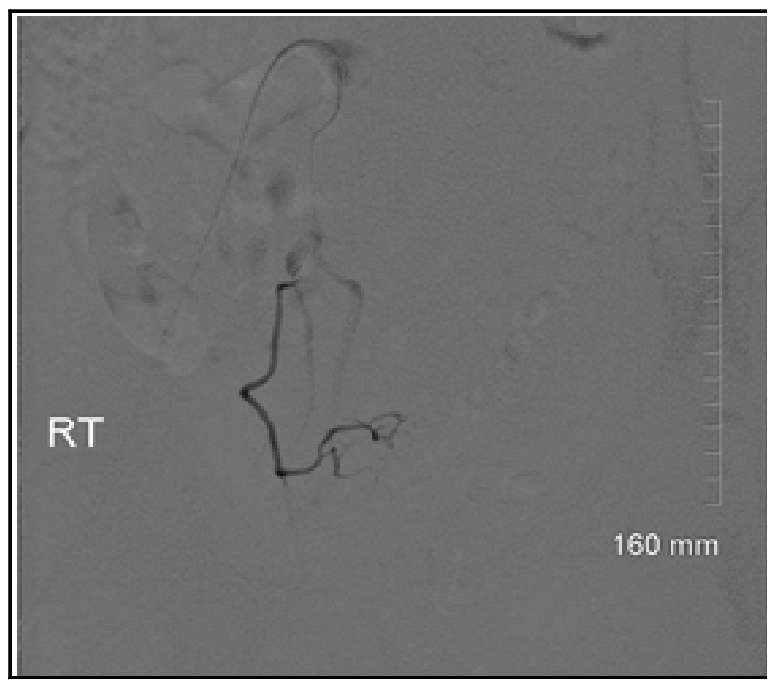

Figure 1. Angiogram showing atretic right uterine artery.

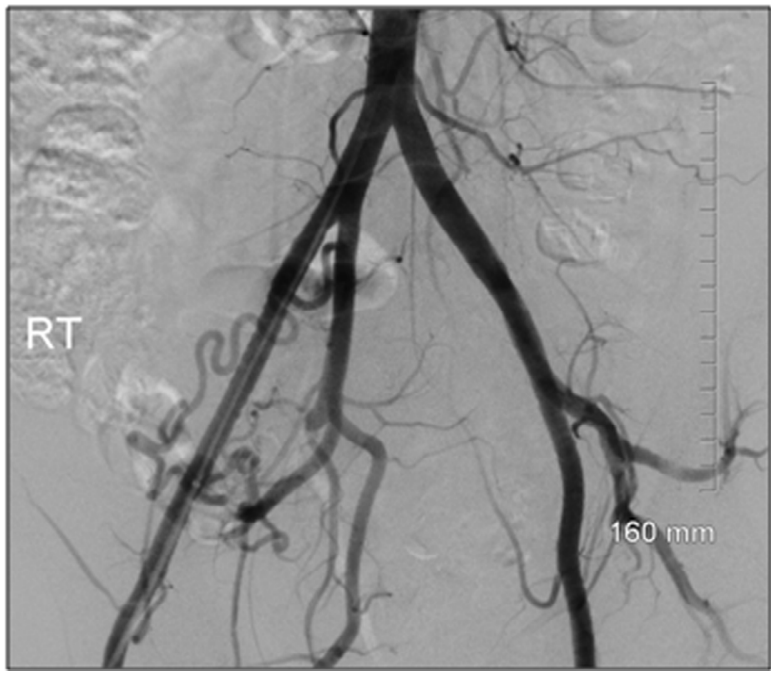

Figure 2. Pelvic angiogram confirming enlarged right ovarian artery which appears to arise from the iliac artery instead of the expected origin from the abdominal aorta just below the right renal artery.

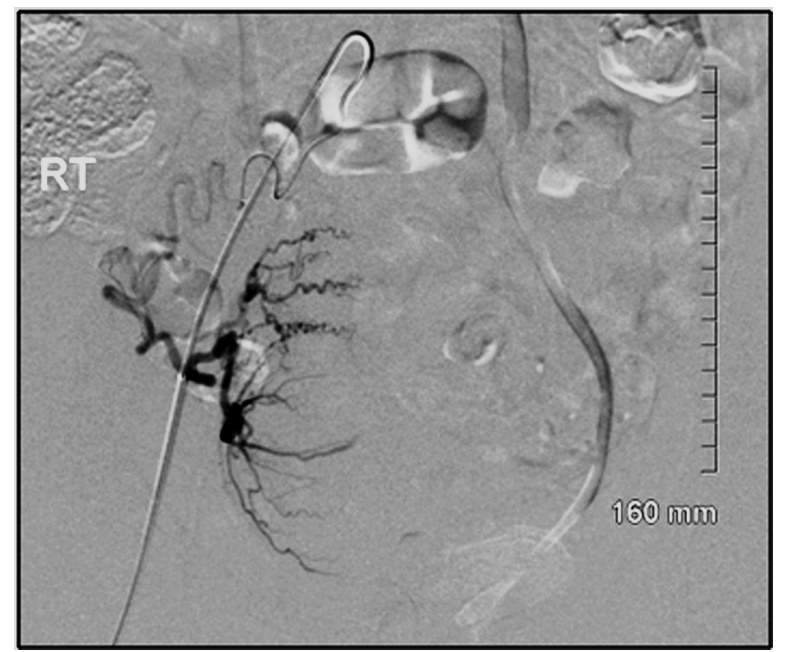

Figure 3. Selective right ovarian arteriogram demonstrates significant flow to the uterus.

her menstrual periods had dramatically improved and her anemia has resolved. She remains asymptomatic at two and a half month follow up.

\section{Discussion}

UAE has been accepted as an effective and less invasive treatment option for women with symptomatic uterine fibroids. Although technical success rates approach $100 \%$, clinical success rates vary from $85 \%$ - 94\% [1-4]. One cause of clinical failure has been shown to be ovarian artery collateral supply to the fibroids [9]. Ovarian artery supply to the uterus has been shown to occur in $11 \%$ of patients who undergo UAE [6,10]. Enlarged ovarian arteries that provide collateral supply to fibroids tend to occur in women with absent or small uterine arteries. In this situation ovarian artery embolization has been shown to increase the likelihood of complete fibroid infarction and clinical success [5].

Although the ovarian arteries typically arise from the abdominal aorta below the level of the renal arteries, several variations to the location of ovarian artery origin have been reported. The reported variants include ovarian artery origin from the renal arteries, the suprarenal abdominal aorta, the inferior mesenteric arteries and the external iliac arteries.

In our patient there was an atretic right uterine artery and an enlarged right ovarian artery with a rare variant origin from the proximal internal iliac artery. There was significant collateral flow from this artery to the uterus and fibroids. We felt that embolization was necessary to optimize the chances of complete fibroid necrosis and clinical success.

The physiologic impact of ovarian artery embolization is not entirely known. There is risk of premature ovarian failure but several studies have shown that the chance of 
embolization induced menopause is no greater in women who had ovarian artery embolization at the time of UAE compared to women treated with UAE alone [11,12].

\section{Conclusion}

Physicians who perform UAE should realize the importance of potential collateral arterial supply from the ovarian artery to the uterus and should be familiar with the variations in ovarian artery anatomy.

\section{REFERENCES}

[1] S. C. Goodwin, B. McLucas, M. Lee, G. Chen, R. Perrella, S. Vedantham, et al., "Uterine Artery Embolization for the Treatment of Uterine Leiomyomata Midterm Results,” Journal of Vascular and Interventional Radiology, Vol. 10, No. 9, 1999, pp. 1159-1165. doi:10.1016/S1051-0443(99)70213-7

[2] G. Pron, J. Bennett, A. Common, J. Wall, M. Asch, K. Sniderman, et al., "The Ontario Uterine Fibroid Embolization Trial. Part 2. Uterine Fibroid Reduction and Symptom Relief after Uterine Artery Embolization for Fibroids," Fertility and Sterility, Vol. 79, No. 1, 2003, pp. 120-127. doi:10.1016/S0015-0282(02)04538-7

[3] I. Pinto, P. Chimeno, A. Romo, L. Paul, J. Haya, M. A. de la Cal, et al., "Uterine Fibroids: Uterine Artery Embolization versus Abdominal Hysterectomy for Treatment-A Prospective, Randomized, and Controlled Clinical Trial," Radiology, Vol. 226, No. 2, 2003, pp. 425-431. doi:10.1148/radiol.2262011716

[4] J. B. Spies, S. A. Ascher, A. R. Roth, J. Kim, E. B. Levy and J. Gomez-Jorge, "Uterine Artery Embolization for Leiomyomata," Obstetrics and Gynecology, Vol. 98, No. 1, 2001, pp. 29-34. doi:10.1016/S0029-7844(01)01382-5

[5] M. M. Barth and J. B. Spies, “Ovarian Artery Embolization Supplementing Uterine Embolization for Leiomyomata," Journal of Vascular and Interventional Radiology, Vol. 14, No. 9, 2003, pp. 1177-1182. doi:10.1097/01.RVI.0000085772.71254.CF
[6] J. P. Pelage, O. Le Dref, P. Soyer, D. Jacob, M. Kardache, H. Dahan, et al., "Arterial Anatomy of the Female Genital Tract: Variations and Relevance to Transcatheter Embolization of the Uterus," American Journal of Roentgenology, Vol. 172, No. 4, 1999, pp. 989-994.

[7] D. L. Smoger, V. Kancherla and R. D. Shlansky-Goldberg, "Uterine Fundal Blood Supply from an Aberrant Left Ovarian Artery Originating from the Inferior Mesenteric Artery: Implications for Uterine Artery Embolization,” Journal of Vascular and Interventional Radiology, Vol. 21, No. 6, 2010, pp. 941-944. doi:10.1016/j.jvir.2010.01.043

[8] J. H. Kwon, M. D. Kim, K. H. Lee, M. Lee, M. S. Lee, J. Y. Won, et al., “Aberrant Ovarian Collateral Originating from External Iliac Artery during Uterine Artery Embolization," Cardiovascular and Interventional Radiology, 2012. doi:10.1007/s00270-012-0406-0

[9] M. Matson, A. Nicholson and A. M. Belli, “Anastomoses of the Ovarian and Uterine Arteries: A Potential Pitfall and Cause of Failure of Uterine Embolization," Cardiovascular and Interventional Radiology, Vol. 23, No. 5, 2000, pp. 393-396. doi:10.1007/s002700010090

[10] A. W. Horton, U. Patel and A. M. Belli, “An Unusual Arterial Supply to the Uterus. A Case Report and Review of Anatomy-Implications for Uterine Artery Embolization,” Clinical Radiology, Vol. 65, No. 12, 2010, pp. 10381042. doi:10.1016/j.crad.2010.07.007

[11] N. N. Hu, D. Kaw, M. F. McCullough, H. Nsouli-Maktabi and J. B. Spies, "Menopause and Menopausal Symptoms after Ovarian Artery Embolization: A Comparison with Uterine Artery Embolization Controls," Journal of Vascular and Interventional Radiology, Vol. 22, No. 5, 2011, pp. 710-715. doi:10.1016/j.jvir.2011.01.441

[12] C. Scheurig-Muenkler, A. Lembcke, V. Froeling, M. Maurer, B. Hamm and T. J. Kroencke, "Uterine Artery Embolization for Symptomatic Fibroids: Long-Term Changes in Disease-Specific Symptoms and Quality of Life,” Human Reproduction, Vol. 26, No. 8, 2011, pp. 2036-2042. doi:10.1093/humrep/der170 